\title{
Self-repairing and tribological behaviour of steel-steel friction pairs lubricated with an oil with magnesium silicate hydrosilicate as additive
}

\author{
Libo Wang, Yi Yang, Gang Yang, Guohong Shui, and Mingxia $\mathrm{Wu}^{*}$ \\ School of Manufacturing Science and Engineering, Sichuan University, Chengdu 610065, PR China
}

Received: 4 September 2018 / Accepted: 17 December 2018

\begin{abstract}
Magnesium silicate hydrosilicate (MSH) was added into a lubricating oil as an additive for testing self-repairing capability of a friction pair when it is lubricated. A systemic examination of the structure and chemical composition of the surfaces with self-repairing layers was carried out with an expectation of reducing the wear and even fully repairing the surfaces through the newly grown layers. The wear behaviour of the surfaces, including that having the deposited self-repairing layers, was examined with reference to the lubricated steel friction pairs, being loaded under different working conditions. The treated surfaces and their chemical composition were examined using scanning electron microscopy (SEM) and EDS/XRD, respectively. The results showed that the level of the concentration of the additive in the lubricating oil does not affect the chemical composition of the self-repairing layer formed, and lubricating oil with $1.0 \% \mathrm{MSH}$ is an optimal concentration value for reducing friction and wear for the friction pairs examined. At the same time, the grown self-repairing layers become more uniform as the duration of sliding between the two parts in a friction pair increases.
\end{abstract}

Keywords: Magnesium silicate hydrosilicate (MSH) / lubricating oil / self-repairing layer / wear behaviour

\section{Introduction}

Metal abrasion is one of three main causes to the failures of machine parts. There are many methods existing that help to reduce wear [1-6], such as rationalization of the structural designs, using lubricants, surface treatments, including coatings. Each of these methods has its own merits and limitations. For more than a decade, the mineral lubricating oil containing natural serpentine has been used effectively as a lubricant, abrasion deterrent and abrasion resistant, and it has exhibited potential to combat wear through self-repairing behaviours [7-12]. A friction pair comprises two rubbing surfaces, defined largely by their composition and topography. Self-repairing is a process in which attrition is automatically compensated by restoration of the original surface geometry through newly growing layer, thus prolonging the life of the working parts. It is realized by chemical-metallurgical bonding between the structural surface and constituents of a lubricant. The tribological behaviour of the friction pairs with ongoing self-repairing can be considered as a result of competition between the rate of the formation of a surface film and its attrition [13].

\footnotetext{
* e-mail: wumingxia@scu.edu.cn
}

Yang et al. reported that a self-repairing layer of 3-6 $\mu \mathrm{m}$ thickness was formed on a 1045 medium carbon steel to steel friction pair, when $0.1 \% \mathrm{MSH}$ was added to the base-oil lubricant and it exhibited the excellent performance as the low friction coefficient was observed. Furthermore, hardness of the layers with MSH was twice higher than that of the 1045 steel [14]. Xu et al. used TEM to analyze the structure and tested nanomechanical properties of the areas near and far away from the embedded particles [15]. Qi et al. used the heat-treated nanoscale serpentine power as additive to the base oil and proposed a model for predicting the film formation [12]. From the narrative it appears that the addition of MSH to the base oil could not only improve the friction and wear properties of the steel-steel friction pairs but also could have a significant effect on the cast iron friction pair [16].

Achieving self-repairing in a friction pair when the special additive is added into a base oil depends significantly on the working conditions under which the friction pair works. For example, in the case of the medium carbon steel and base oil without the additive, the wear mechanism changes from slight abrasion to severe abrasion when the load is increased. Although, after MSH is added, the tribological behaviour is still influenced by the loading condition, existence of the powder agglomeration could help to change the condition from sliding friction to rolling 

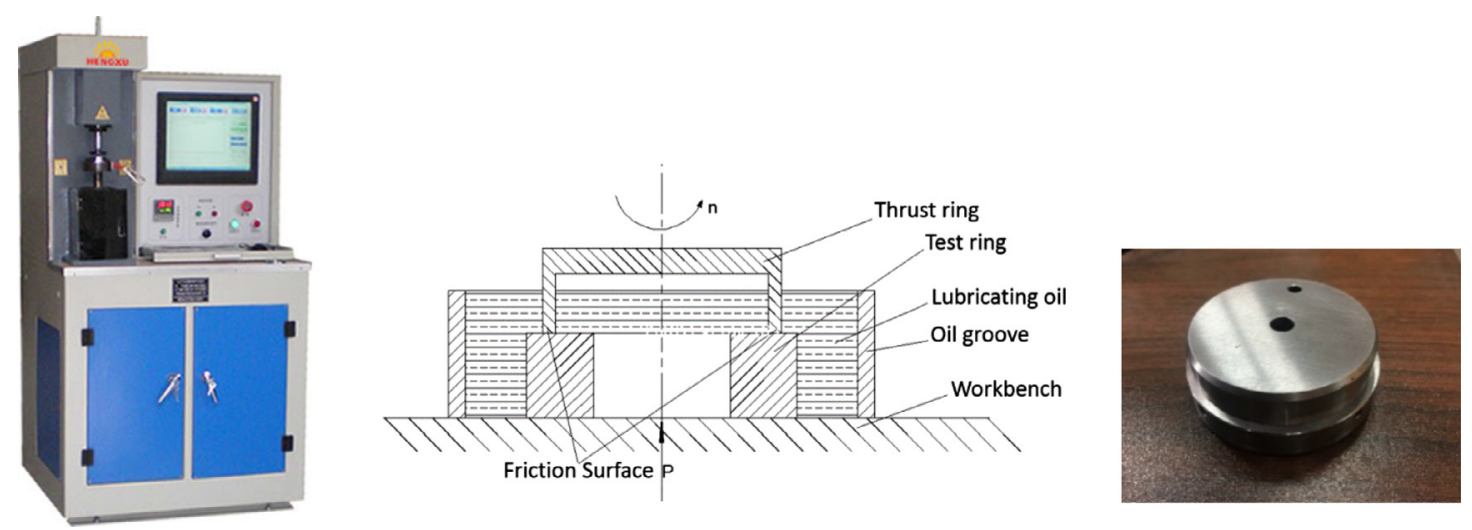

Fig. 1. Experimental apparatus and photo of a sample produced: (a) Equipment, (b) Illustration of the working principle of the lubrication/friction test and (c) Sample part.

friction and thus reduce effective friction coefficient, when the loading condition was lighter [17]. After the phase transition of the MSH took place, the surface of the sample would be flecked and sticky, and it would, however, still exhibit the excellent performance [18].

Although achieving self-repairing functionalities through using lubricating oils with MSH additive has been reported by several researchers, these have been carried out mostly based on individual cases concerning this topic [19-21]. In a previous study, Huang tested hardness of the self-repairing layers that were produced when natural serpentine was added into a lubricating oil, and the bonding between the layers and the substrate was also analyzed [11]. The study reported in this paper is a systemic examination of the structure and chemical composition of the surfaces with self-repairing layers that were generated recently, when MSH was added into the lubricating oil. It was expected that through this study, more comprehensive understanding of the formation mechanism of the self-repairing layers and hence, application conditions, could be established. In this study, friction tests using a 1045 medium carbon steel to steel friction pair with No. 32 base lubricating oil containing MSH additive were conducted. The effects of different concentrations of the additive and the effect of wear time on the anti-wear effect were investigated. The mechanism of the selfrepairing layer growth was analyzed. These are described and discussed in this article. In particular, the relationship between the mass change and generation of the selfrepairing layers are discussed in great depth, in the article, aiming at further clarifying some issues around the mechanisms responsible for the excellent tribological properties of the treated surfaces, when MSH as additive is used.

\section{Test apparatus, material and experimental procedure}

\subsection{Experimental apparatus, materials and method}

The steel-steel friction pairs of 1045 medium carbon steel were used for the experiment. The samples were sectioned and the surface was polished after annealing. The surface hardness of the samples was $20 \mathrm{HRC}$. The concentration of MSH in the No. 32 mineral base oil was varied between the tests, being $0,1.0,2.0$ and $4.0 \%$, respectively. The particle size of the MSH was about $5 \mu \mathrm{m}$. No. 32 mineral base oil as the base oil has kinematic viscosity value between $28.8-35.2 \mathrm{~mm}^{2} / \mathrm{s}$ at $40^{\circ} \mathrm{C}$.

The experimental apparatus used is illustrated in Figure 1. Essentially, it consists of a cylindrical container with a hollow cylinder (test ring) made from the test material placed along its axis. A thrust ring with the same test material, located along the same axis, was placed on the hollow cylinder, while rotating, to form a friction pair. The rubbing surfaces were completely immersed in the lubricating medium. The total testing time was $16 \mathrm{~h}$. In the first $8 \mathrm{~h}$, the contact load between the friction pair was $400 \mathrm{~N}$ and the rotational speed $2.26 \mathrm{~m} / \mathrm{s}$. For the following $8 \mathrm{~h}$, the load was $100 \mathrm{~N}$ and the rotational speed was $0.56 \mathrm{~m} / \mathrm{s}$.

To determine the effect of the working time, two 40-h long tests were undertaken with MSH concentrations of $4.0 \%$. To eliminate the artificial effect, two samples were cut randomly from the specimens by electric spark wire cutting for each case, and the surface was further cleaned with the alcohol solution for $30 \mathrm{~min}$ and dried. The weighing method was used, first, to roughly assess the existence of layers.

For simplicity, four test modes were defined as follows: A - friction pairs without additive; $\mathrm{B}$ - friction pairs with $1.0 \%$ additive; $\mathrm{C}-$ friction pairs with $2.0 \%$ additive; $\mathrm{D}-$ friction pairs with $4.0 \%$ additive, while $\mathrm{A} 1, \mathrm{~B} 1, \mathrm{C} 1$ and $\mathrm{D} 1$ represent the friction coefficient corresponding to concentration of $0,1.0,2.0$ and $4.0 \%$, respectively.

\subsection{Surface characterization}

The topography and chemical composition of the friction surfaces were obtained using SM-7500-F SEM (containing EDS) and XRD-7000, respectively. The Raman spectrum of the developed self-repairing layers was further examined using a HORIBA XploRA PLUS Raman spectrometer, and morphology and composition were further checked using an 


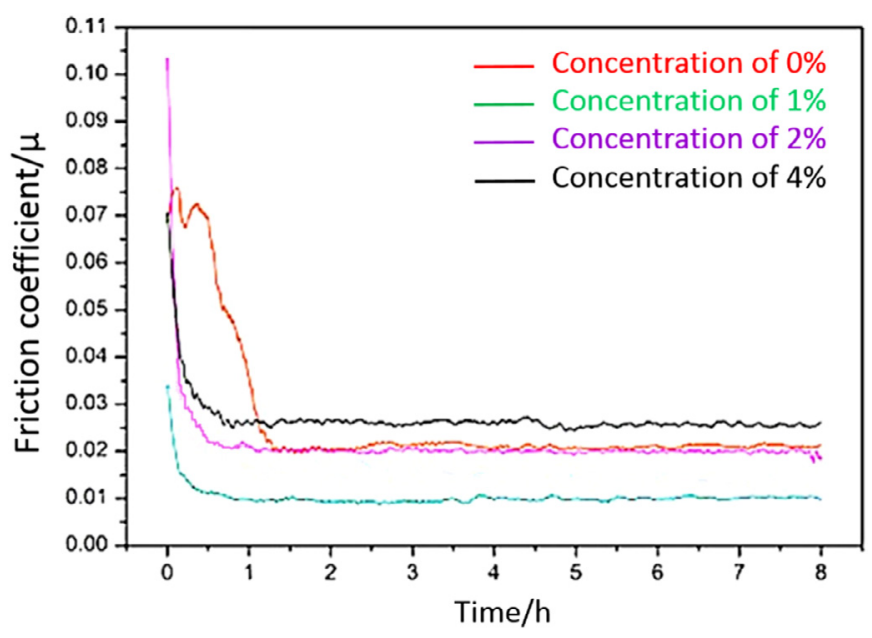

Fig. 2. Relation of friction coefficient to the time of testing (1045 steel friction pair, oil-lubricated with different concentrations of MHS additive).

OLYMPUS GX51 metallographic microscope and X'Pert Pro MPD X-ray with a spectrometer (EDS) diffractometer, respectively. In addition, Vickers hardness was measured using a HVS-1000 digital display instrument.

\section{Results and discussion}

\subsection{Effects of the MHS concentration}

\subsubsection{Coefficient of friction}

Figure 2 shows how friction coefficient varies with testing time, for different concentrations of MSH. It can be seen that the friction coefficient monotonously increases with the MSH concentration.

Within less than $1.5 \mathrm{~h}$, after a period of instability, friction for condition A1 decreases rapidly from a value of about 0.07 and then remains at a nearly constant value of about 0.02 for the remaining time. For the B1 condition, in less than $1.5 \mathrm{~h}$, friction reduces rapidly and smoothly from an initial value of about 0.07 to a steady-state value of about 0.01. For the $\mathrm{C} 1$ condition, in about the same time as for condition B1, friction reduced from an initial uniquely high value of about 0.07 to a steady-state value of about 0.02 , being the same value as for the $\mathrm{A} 1$ condition. Friction reduction for D1 condition is similar to that for B1 condition but the steady-state value, at about 0.025 , is highest of all.

When the concentration is low, there are fewer particles in the lubricating oil. During the experiment, the particles bear the high contact stress between the friction surfaces. They were shattered to the uniform sizes, and gradually became round shaped particles. As a consequence, sliding friction is gradually transformed into rolling friction, indicting a lower effective friction coefficient [10]. The higher the concentration of MSH, the more the particles involved, which bears the load between relatively moving surfaces, and hence these are less ground, resulting in the less changes in shapes. Since the particles have large surface energy, the agglomeration will become significant, and this is why the friction coefficient in condition $\mathrm{D}$ was much higher than that in others.

\subsubsection{Wear of the friction surfaces}

Unlike traditional liquid lubricating conditions, when a lubricating oil contains MSH, self-repairing layers will grow on the friction surfaces when the local thermodynamic condition is met. The growing repairing layer will compensate for the losses due to wear, to some extent. Therefore, the total wear is determined by a dynamic balance between the wear rate and the thin film growth rate during the two combined processes.

The variations of the weight of the test ring and thrust ring, shown in Figure 3, indicate the weight losses of the two rings as well their changes. For the condition A, wear of the test ring is greater than the thrust ring at the $16 \mathrm{~h}$ in the experiment for $\mathrm{A}$. In the lubricating oil containing selfrepairing additives $(\mathrm{B}, \mathrm{C}, \mathrm{D})$, the wear of the thrust ring is higher than that for the test ring. From the experiment, it is shown that the concentration of MSH does not affect the trend of the change of the weight of the thrust ring, but under the influence of different concentrations of MSH, the amount of wear is different. When the concentration was $1.0 \%$, the wear of the thrust ring has a minimum value, and it was decreased by $24.4 \%$, compared to that for the condition A. When the additive concentration reached 2.0 and $4.0 \%$, the wear volume of the thrust ring increased by 69.3 and $31.6 \%$, respectively, compared to that for condition A. The results given in Figure 3, nevertheless, do not fully reflect the actual trend of the material weight changes before $8 \mathrm{~h}$ of the tests, since the samples measured were ones that were taken from the $8 \mathrm{~h}$ of the tests.

For all the four concentrations of MSH tested, the thrust ring loses its weight at the end, and the test ring, however, may gain some weight after going beyond the lowest point of the weight change, although both rings were made from medium carbon steel. During the tests, the thrust ring was kept rotating and the test ring fixed. The lubricant flows over the thrust ring surface and test ring surface are different: the relative speed of the lubricant flow over the thrust ring surface is faster than that over the test ring surface, which results in advantageous conditions for more particles adhered to the test ring surface. While the pressurisation is forcing these particles to penetrate into the test ring surface, it is also accompanied with temperature increase and possibly a phase change of the $\mathrm{MSH}$.

\subsubsection{Friction surfaces}

Figure 4 shows surfaces of the specimens tested with different additive concentrations after a 16 -h test. The scratches are marked by black arrows and ploughing by white ones. Three samples were taken randomly from the three test rings subjected to $\mathrm{B}, \mathrm{C}$ and $\mathrm{D}$ condition, respectively. Self-repairing layers were observed in the first and third sample, these corresponding to the working condition B and D, respectively. 

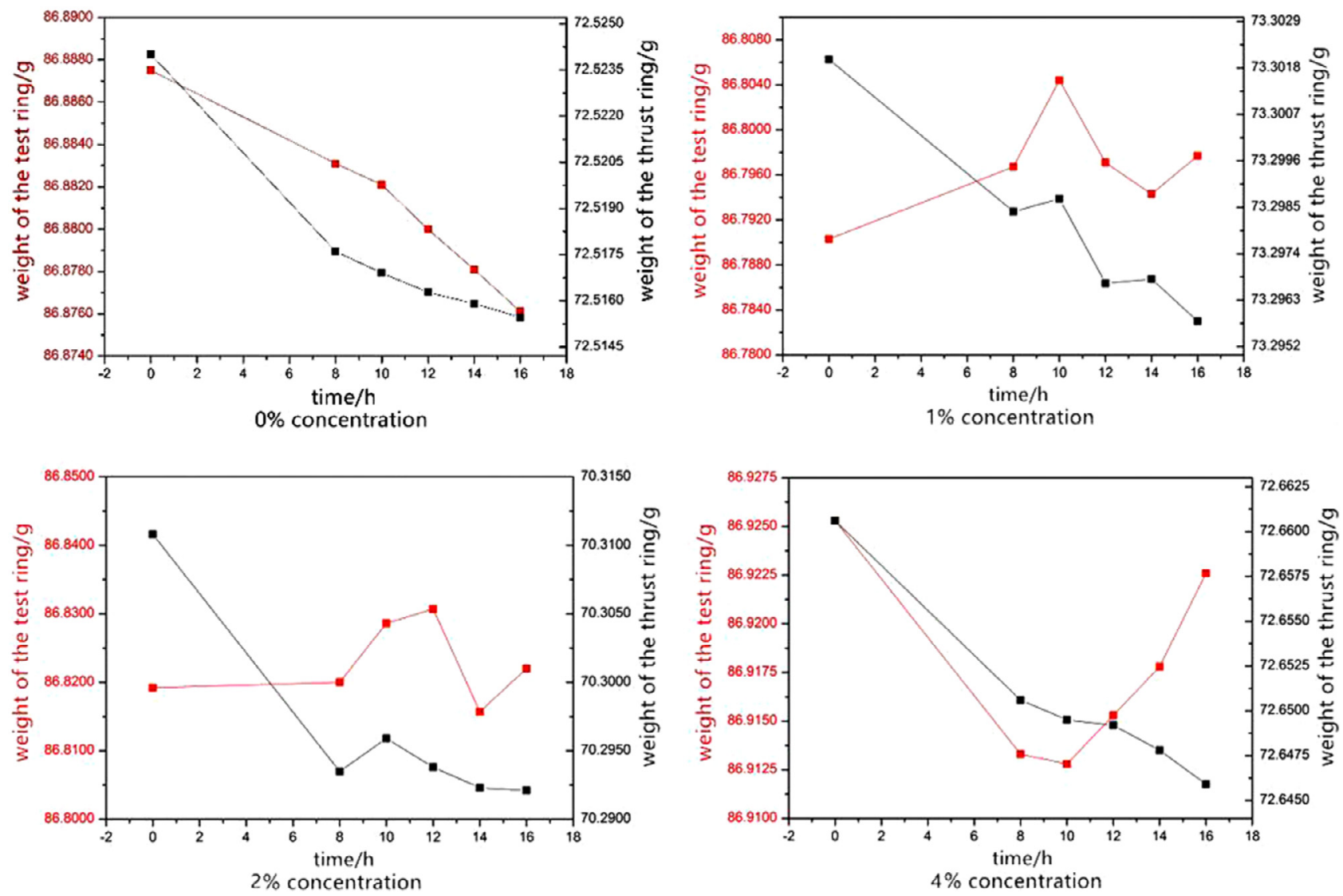

Fig. 3. Effect of concentration of MHS additive on the metal weight variations (the red line and the black line represent the weight of the test ring and the thrust ring, respectively).
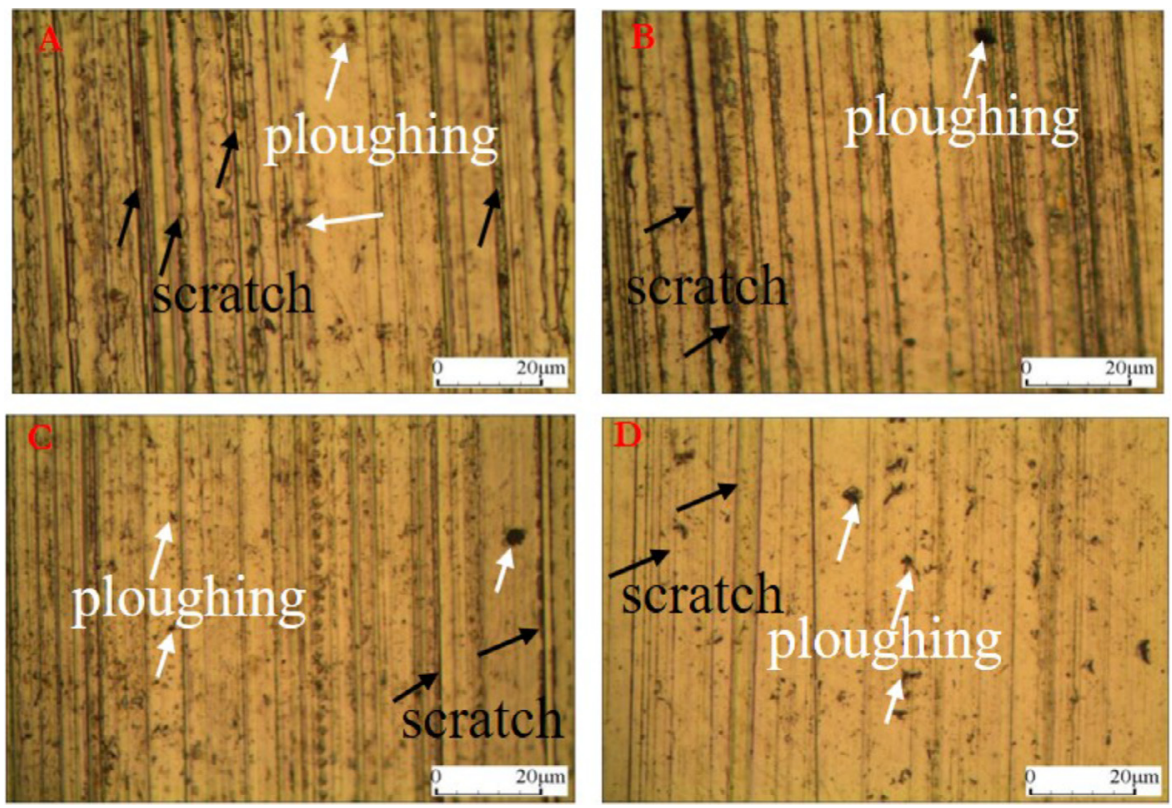

Fig. 4. Images of the surfaces of the specimens tested with different additive concentrations (16 h testing). 
Table 1. Summary of the surface topography state corresponding to conditions A, B, C and D.

\begin{tabular}{llll}
\hline Concentration & Surface scratch & Ploughing & Other phenomenon \\
\hline $0 \%$ & Many & Many and deep & Local spalling \\
$1.0 \%$ & Obvious & Many and deep & Local spalling \\
$2.0 \%$ & Obvious & Few and shallow & Wide-range spot \\
$4.0 \%$ & Few & Few and shallow & Local spalling \\
\hline
\end{tabular}
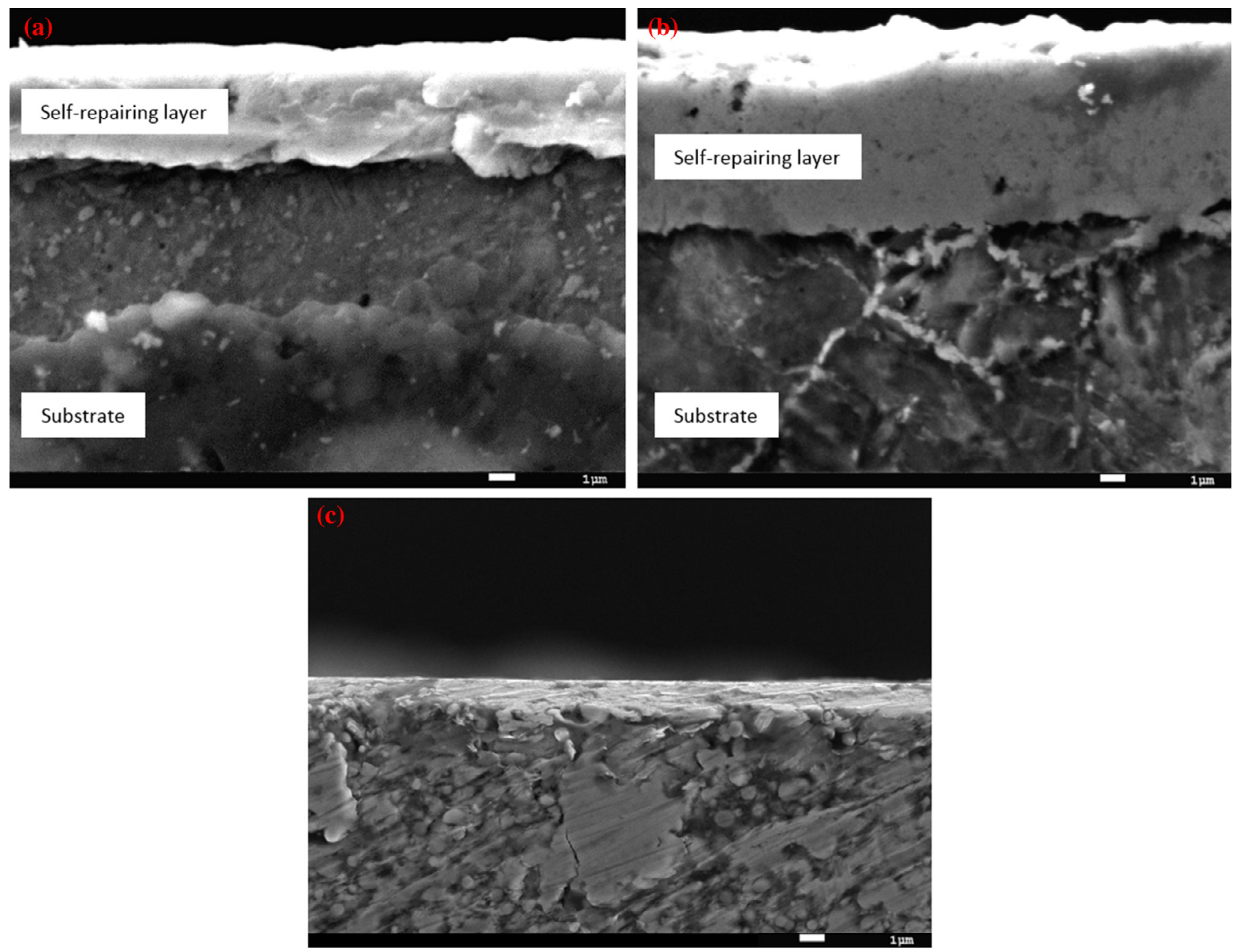

Fig. 5. SEM images of deposited layers with concentration of D (a), B (b) and A (c) for a test duration of $16 \mathrm{~h}$.

The thickness of the repairing layer was about 3-4 $\mu \mathrm{m}$ and uneven, and there was no repairing layer created in some regions. Although it was seen that the third test ring had lost weight in total, it still had self-repairing layer on it, indicating that the total wear rate was larger than the rate of formation of the self-repairing layer. Also, it is clear that the mass change alone cannot be a direct indicator showing existence of a self-repairing layer, and the combined effect of the wear rate and the film-forming rate is a key factor to determine the final state of the wear.

Table 1 summarizes the state of the surface topography obtained from the samples corresponding to the working conditions A, B, C and D, respectively. The results suggest that there must be an optimum condition existing for those friction pairs, being influenced strongly by the additive concentration of MSH, initial roughness of the surfaces, type of the materials, rotational speed of the thrust ring and pressure applied.

Figure 5 shows two self-repairing layers formed (silvery-white part), and these are dense coatings bonded well to the substrate. An examination of chemical compositions in the areas E, F, E1 and F1 showed that the substrate and self-repairing layers have a similar chemical composition and contain elements of $\mathrm{C}, \mathrm{O}, \mathrm{Si}, \mathrm{Cr}$, 
Table 2. The chemical composition in different samples.

\begin{tabular}{llllllll}
\hline Regional element & $\mathrm{C}$ & $\mathrm{O}$ & $\mathrm{Si}$ & $\mathrm{Cr}$ & $\mathrm{Mn}$ & $\mathrm{Fe}$ & Total \\
\hline E area/wt.\% & 4.51 & 2.38 & 0.57 & 0.16 & 0.48 & 91.90 & 100.00 \\
F area/wt.\% & 0.42 & 1.32 & 0.17 & 0.21 & 0.52 & 97.36 & 100.00 \\
E1 area/wt.\% & 4.63 & 3.82 & 0.61 & 0.19 & 0.45 & 90.30 & 100.00 \\
F1 area/wt.\% & 0.45 & 1.36 & 0.25 & 0.17 & 0.58 & 97.19 & 100.00 \\
\hline
\end{tabular}

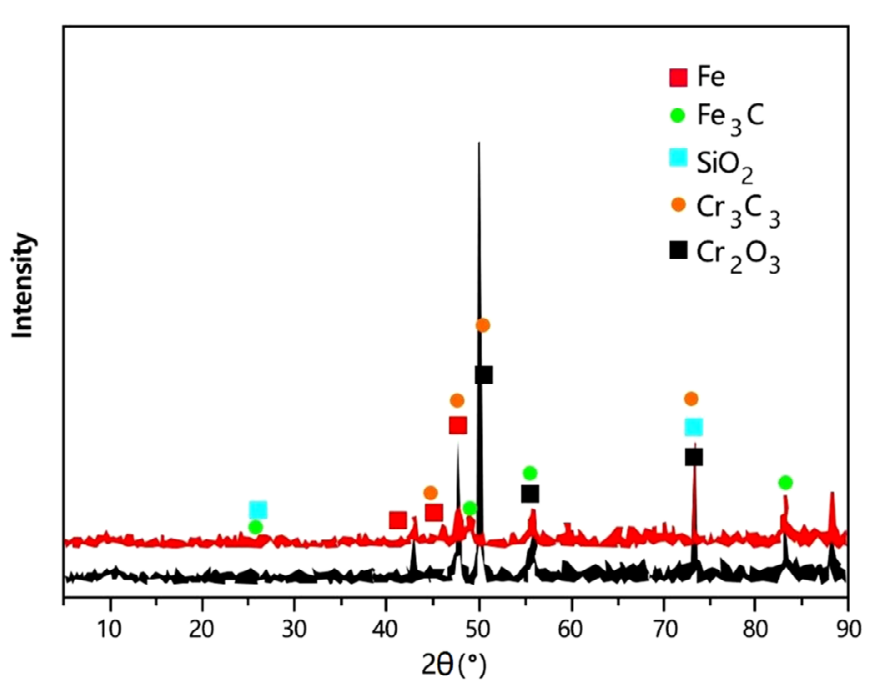

Fig. 6. Results from the XRD analysis of layers corresponding to the condition B (red curve) and condition D (black curve).

$\mathrm{Mn}$ and Fe. The characteristic element of $\mathrm{Mg}$ was not found in the layer. The content of each element in the substrate and the repairing layer has some difference. As shown in Table 2, from the surface to the substrate, the amount of C, $\mathrm{O}$ and $\mathrm{Si}$ reduced, while $\mathrm{Fe}, \mathrm{Cr}$ and $\mathrm{Mn}$ increased. The $\mathrm{C}$ and $\mathrm{O}$ in the repairing layers are higher than that of the steel substrate. The $\mathrm{C}$ element may be from the carbonization of the lubricating oil and the $\mathrm{O}$ may be due to the phase change of MSH.

Figure 5 shows that although the self-repairing layer for condition $\mathrm{B}$ was thicker than that for condition $\mathrm{D}$, the layer generated for condition $\mathrm{D}$ is denser than that for condition $\mathrm{B}$, and there is no self-repairing layer on the rubbing surface under condition $\mathrm{A}$, as shown in Figure $5 \mathrm{c}$. It can be explained that a self-repairing layer is initiated at localized areas where a high energy level exists, and it is later expanded into other areas, during which the layer may experience both alternating mechanical and thermal stresses, leading to fatigues of the surface/layer and new fracturing. Depending on the local conditions, including initial material surface conditions, the layers with different morphologies may exist, although these, basically, are similar, in terms of the type of chemical composition and proportions of the chemical elements. During the experiment for condition D, a higher energy level was required, and correspondingly, the surface suffered more from the abrasive wear.

\subsection{XRD analysis}

It can be seen, from Figure 6, that the self-repairing layers contain $\mathrm{Fe}_{3} \mathrm{C}$ and other phases, such as $\mathrm{SiO}_{2}$, $\mathrm{Cr}_{7} \mathrm{C}_{3}$ and $\mathrm{Cr}_{2} \mathrm{O}_{3}$, resulting in their strength and hardness. The major element of MSH, Mg, is not evident. Due to high temperatures and pressures at the friction interface, the MSH releases highly reactive oxygen due to its phase change, which activates a redox reaction. $\mathrm{SiO}_{2}$ is the phase transition product of MSH, and becomes a significant second phase reinforcement in self-repairing layers.

\subsection{Raman analysis}

The region of the repairing layer was further examined by Raman analysis (Fig. 7). The wave length was $532 \mathrm{~nm}$ and the displacement between 500 and $2000\left(\mathrm{~cm}^{-1}\right)$. The peak corresponding to $658 \mathrm{~cm}^{-1}$ is $\mathrm{Fe}_{3} \mathrm{O}_{4}$, and $1322 \mathrm{~cm}^{-1}$ (D-bond) is near the peak of DLC $\left(1360 \mathrm{~cm}^{-1}\right)$. It is the symbol of sp $\mathrm{sp}^{3}$ bond. Also, the peak of $1598 \mathrm{~cm}^{-1}$ (close to $1580 \mathrm{~cm}^{-1}$, "g-bond") corresponds to sp ${ }^{2}$ bond. ID $/ \mathrm{IG}=0.8$. This result indicates that complex carbon-containing substances exist in the surface of the repairing layer.

It should be noted that $\mathrm{Fe}_{3} \mathrm{O}_{4}$ was not detected by the XRD analysis for a depth of several micrometers. Raman analysis essentially deals with surfaces. Thus, it is implied from the results that $\mathrm{Fe}_{3} \mathrm{O}_{4}$ is mainly accumulated in a thin self-repairing layer.

\subsection{Surface morphology}

In order to further examine the formed microstructure, AFM images for a self-repairing layer was obtained to determine its three-dimensional topography. This is shown in Figure 8. The image is from an area $20 \mu \mathrm{m}$ $\times 20 \mu \mathrm{m}$ on a specimen tested under condition B. Generally, there are many small pores of $<1 \mu$ m diameter in the layers; these could have been released by the vapour produced by the phase transition of MSH. The largest pore is about $870 \mathrm{~nm}$ deep and about $350 \mathrm{~nm}$ wide, indicating that the self-repairing layer may not be perfectly formed. Figure 8b shows a larger local area than Figure $8 \mathrm{a}$, and the roughness is about $4 \mathrm{~nm}$. Small, submicron sized pores exist in the surface with a maximum depth of about $22 \mathrm{~nm}$. These small pores may act as reservoirs for the lubricating oil during surface rubbing, and some may also penetrate into the deeper section of the repairing layer. 


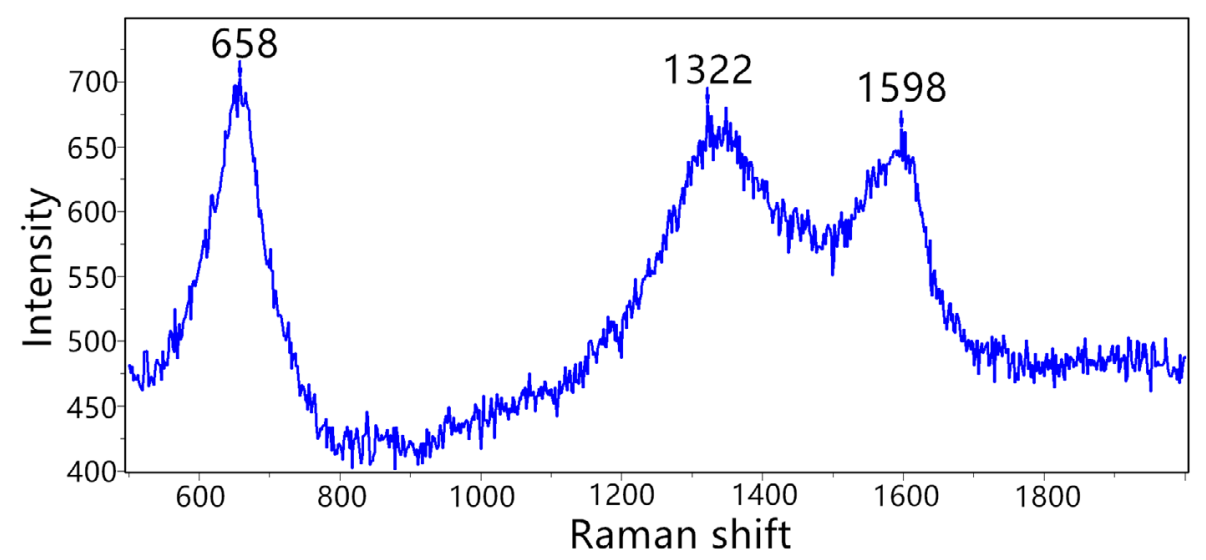

Fig. 7. Raman result.

(a)

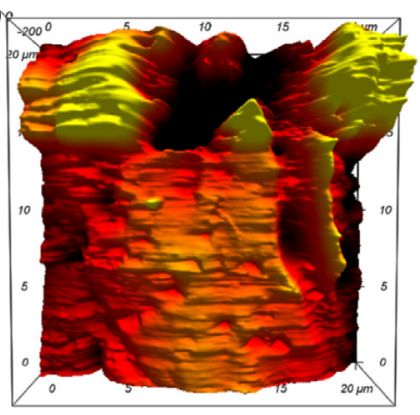

(c)

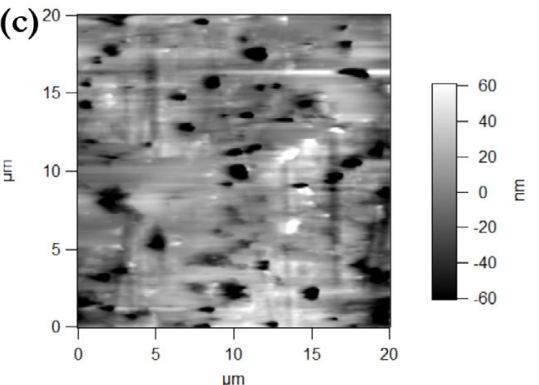

(b)
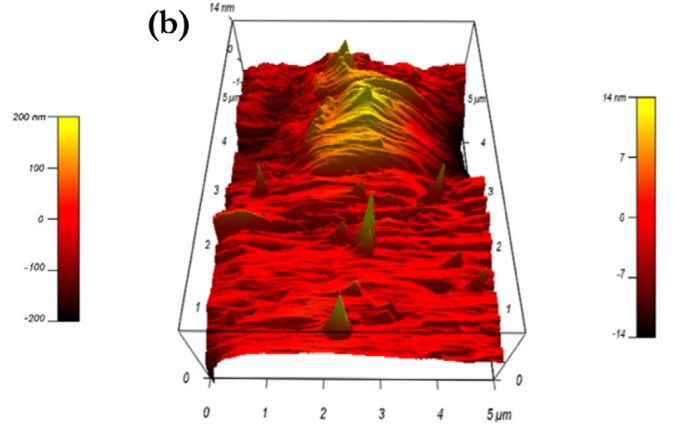

(d)

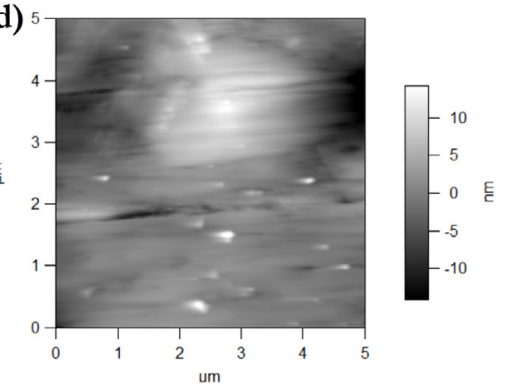

Fig. 8. AFM surface of an area of $20 \mu \mathrm{m} \times 20 \mu \mathrm{m}$ in (a) and (c), and $5 \mu \mathrm{m} \times 5 \mu \mathrm{m}$ in (b) and (d); a surface generated under condition B.

\subsection{Microhardness}

Ten points were randomly selected from a sample produced under the surface condition B for a microhardness test, five being in the self-repairing layers and the others in the substrate material. The load was $50 \mathrm{~g}$ and the pressure maintained for $10 \mathrm{~s}$. The results are presented in Figure 9. These show that the hardness of the repairing layer is significantly higher than that of the substrate material (1092.9 HV, compared to $652.9 \mathrm{HV}$ on average for the substrate). The high hardness of the self-repairing layer indicates a better performance of the surface expected against wear.

\subsection{Working time}

The tribological system behaviours usually change with time, and hence, time is an important factor to be considered when examining interactive phenomenon of the film growth and material loss due to wear, when the MSH additive is used for achieving self-repairing functionalities. To examine this factor, several tests were performed at room temperature for which the MSH additive concentration of $4.0 \%$ was used. Two groups of parameters were pre-set for the experiment: $100 \mathrm{~N}$ load and $0.56 \mathrm{~m} / \mathrm{s}$; and $400 \mathrm{~N}$ load and $2.26 \mathrm{~m} / \mathrm{s}$ on the thrust ring were used. Each test was carried out for up to $40 \mathrm{~h}$ and the thrust ring and test ring were weighed every $4 \mathrm{~h}$, and a small sample was taken from the test ring at every $8 \mathrm{~h}$ (being sectioned from the test ring).

Since a repairing layer, which is deposited on the substrate material, may have a different density than that of the substrate, the overall weight loss is only used for a rough indicator of the total wear. It could also mean that, for instance, wear of the steel could still be compensated by a different volume of the deposited layer; even the weight 


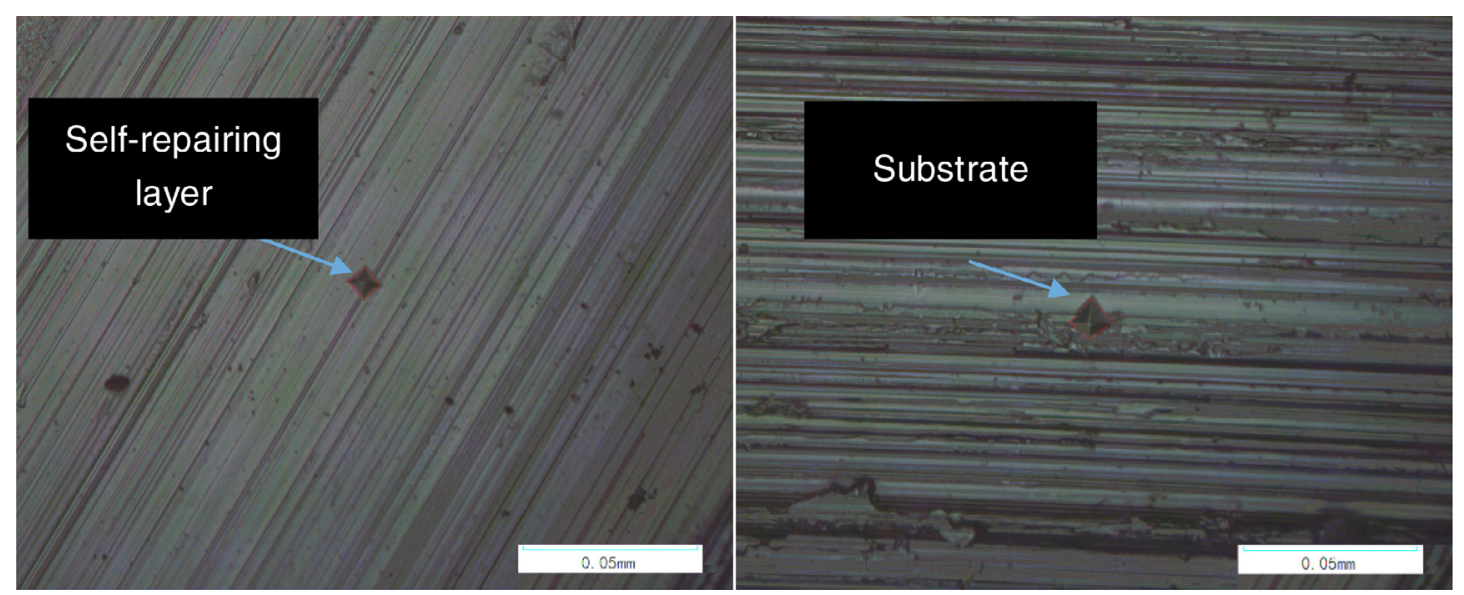

Fig. 9. Microhardness of the B conditioned surface.

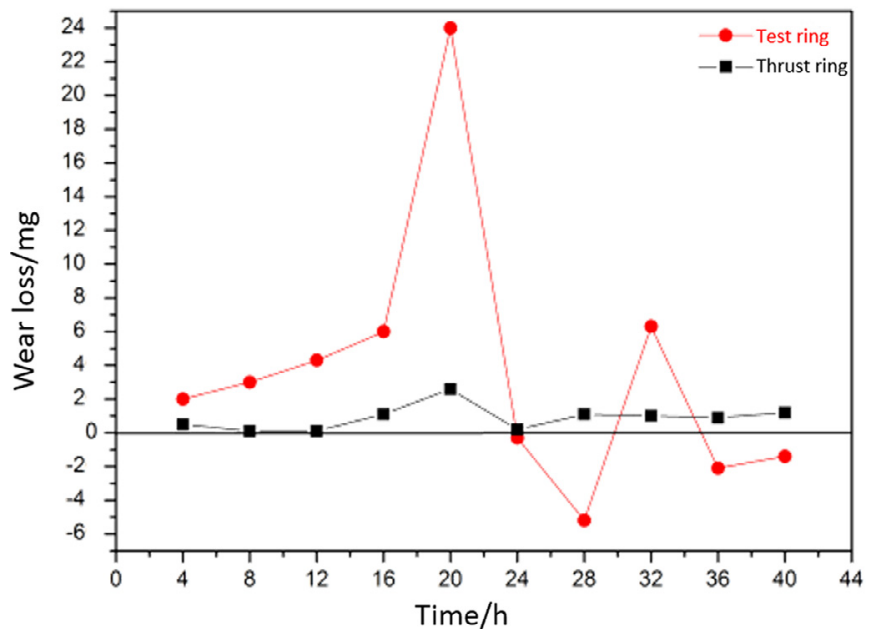

Fig. 10. Relations of weight loss with the test time (load $100 \mathrm{~N}$, rotational speed $0.56 \mathrm{~m} / \mathrm{s}$, and additive concentration $4.0 \%$ ).

loss was measured to be larger than zero, vice versa, depending on the density of the deposited layer material.

Figure 10 shows that the weight loss of the thrust ring increased gradually until the balance was finally maintained. In the first $12 \mathrm{~h}$, the weight loss was almost zero, then, it reached a maximum of $2.6 \mathrm{mg}$ after $20 \mathrm{~h}$. Between about $24-40 \mathrm{~h}$, the weight loss did not change significantly and remained at about $1 \mathrm{mg}$. This is in accord with the traditional friction law.

In the initial $20 \mathrm{~h}$ of testing, wear of the test ring significantly increased with time, to a maximum value of about $24 \mathrm{mg}$. In the following 20 testing h, the wear of the test ring became "negative." At the 28 th $\mathrm{h}$, the "negative wear" had a weight of $5.2 \mathrm{mg}$. At the end of the $40 \mathrm{~h}$ full test, the wear was $-1.4 \mathrm{mg}$. A friction pair could lose or be added the weight with time, depending on the rate of the growth of the repairing layer.

The mass changes measured with time, as shown in Figure 11, indicate an opposite trend for the thrust ring and the test ring. At the end of the experiment, the total

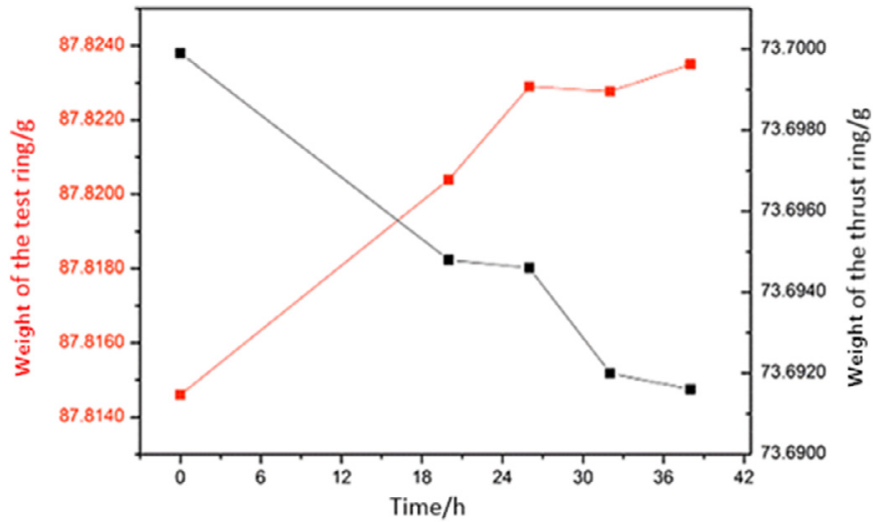

Fig. 11. The mass change of the samples (additive concentration of $4.0 \%$, working time $40 \mathrm{~h}$, thrust force $400 \mathrm{~N}$ and rotating speed $2.26 \mathrm{~m} / \mathrm{s})$.

wear loss of the thrust ring was $8.3 \mathrm{mg}$. The mass of the test ring increased continuously and at the end of the test, increased by $8.9 \mathrm{mg}$. A self-repairing layer with thickness of $4-5 \mu \mathrm{m}$ was observed in the cross section of the test ring.

Figure 12 shows the distribution of the layer generated on the test ring surface for the two working time durations. The self-repairing layer existed mainly in the localized areas; its distribution is uneven and its thickness about $3-5 \mu \mathrm{m}$. The results suggest that prolonging the rubbing time is beneficial to forming a uniform layer.

The self-repairing process is a dynamic process between the wear and film growth. As rubbing time increases, the mechanical friction continuously provides material and energy to the repairing mechanism. Simultaneously, the generated layers are also degraded by the effects of alternating stresses, fatigue ruptures, brittle fractures and corrosive pitting. Nevertheless, as the hybrid process goes on for a sufficient length of time, self-repairing layer thickness will increase, finally, to a desired value and uniformity. 

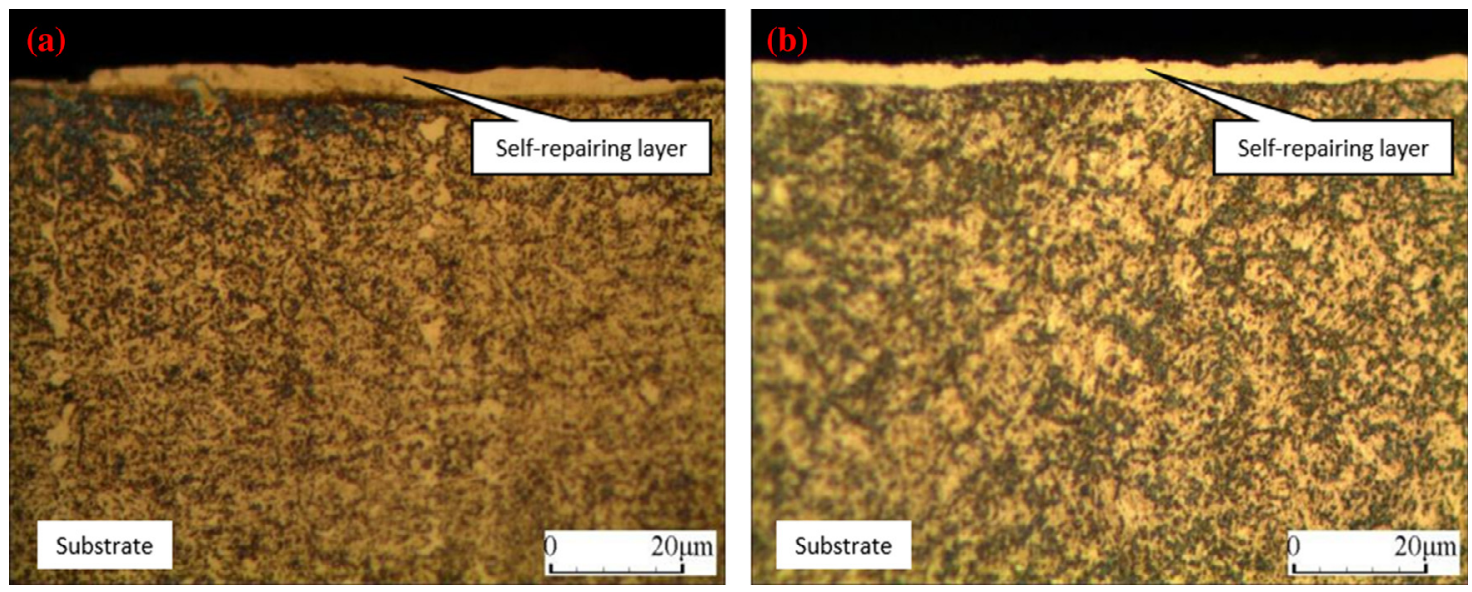

Fig. 12. The distribution of the self-repairing coated layer at the testing time of (a) $16 \mathrm{~h}$, and (b) $40 \mathrm{~h}$, under the condition D.

\section{Conclusions}

A series of experiments using 1045 medium carbon steel friction pairs, lubricated with a base oil with $\mathrm{MSH}$ additive, were undertaken and effects of the MSH concentration and rubbing time on the surface topography and development of self-repairing layers have been newly investigated. The analysis of the results leads to the following conclusions:

(1) Under certain working conditions, MSH can be used as additives to form self-repairing layers for about $3-5 \mu \mathrm{m}$ on the working surface of friction pairs. The hardness of the layers is more than twice that of the substrate. The concentration of MSH in a base oil may not affect the chemical composition of the self-repairing layer formed, but it influences the quality of the layer and the process time required. A concentration of $1.0 \%$ of MSH resulted in lowest friction for the formed surface for the steel-steel pair.

(2) No Mg element was found in the repairing layer deposited, and the chemical composition was similar to that of the 1045 steel substrate, apart from the proportion of $\mathrm{C}$ and $\mathrm{O}$ elements being higher. The elements $\mathrm{C}$ and $\mathrm{O}$ are deemed to be derived from the pyrolysis of the lubricating oil and a phase change of magnesium silicate hydroxide.

(3) The Raman analysis indicated that there is a thin layer mainly made of $\mathrm{Fe}_{3} \mathrm{O}_{4}$ in the self-repairing layer. It may indicate that there are specific sublayers formed in the self-repairing layer, being made of different elements. Further study will be carried out to examine this phenomenon.

(4) Self-repairing layers may be generated randomly over the rubbing surfaces of the friction surface, which cannot be addressed by increasing the load acting on the thrust ring alone. The interface pressure, way of rubbing being conducted, rubbing speed and the time duration of rubbing influence the formation and preservation of the repairing layer. These also indicate that for a particular engineering application, an optimum combination of the material, component surface geometry, additive and concentration in the lubricating oil and the working conditions would be needed if satisfactory self-repairing functionalities are to be achieved.

The funding from the Natural Science Foundation of China (Grand numbers 51575369, 51675357) to support the research reported in this paper is acknowledged.

\section{References}

1. H. Spikes, Tribology research in the twenty-first century, J. Sci. Tribol. Int. 34 (2001) 789-99

2. G.G. Fuentes, E. Almandoz, H.S. Dong, Y. Qin, S. Mato, Vapor deposition technologies for hot forming tools: a review, J. Sci. Manuf. Rev. 1 (2014) 1-17

3. Z.X. Zhang, H.S. Dong, Recent development in low friction and wear-resistant coatings and surfaces for high-temperature forming tools, J. Sci. Manuf. Rev. 1 (2014) 1-12

4. M. Motuku, Parametric studies on self-repairing approaches for resin infused composites subjected to low velocity impact, J. Sci. Smart Mater. Struct. 1 (1999) 623-638

5. L. Rapoport, Y. Feldman, M. Homyonfer et al., Inorganic fullerene-like material as additives to lubricants: structurefunction relationship, J. Sci. Wear 225-229 (1999) 975-982

6. T.S. Chow, Wetting of rough surfaces, J. Sci. Phys. 10 (1998) 445-451

7. Y. Yu, J.L. Gu, Y.F. Kang et al., Surface restoration induced by lubricant additive of natural minerals, J. Sci. Appl. Surf. Sci. 253 (2007) 7549-7553

8. H.L. Yu, Y. Xu, P.J. Shi et al., Tribological behaviours of surface-coated serpentine ultrafine powders as lubricant additive, J. Sci. Tribol. Int. 43 (2010) 667-675

9. Y.S. Jin, S.H. Li, Z.Y. Zhang, In situ mechanochemical reconditioning of worn ferrous surfaces, J. Sci. Tribol. Int. 37 (2004) 561-567

10. Z.N. Jia, Y.L. Yang, J. Chen, Influence of serpentine content on tribological behaviours of PTFE/serpentine composite under dry sliding condition, J. Sci. Wear. 268 (2010) 996-1001

11. H.S. Xie, W.G. Zhou, Y.W. Li, J. Guo, Z.M. Xu, The elastic characteristics of serpentinite dehydration at high temperature-high pressure and its significance, J. Geophys, 43 (2000) 806-811 
12. X.W. Qi, Characterization and auto-restoration mechanism of nanoscale serpentine powder as lubricating oil additive under high temperature, J. Sci. Tribol. Int. 44 (2011) 805-810

13. S.L. Huang, Performance analysis on restoration layer of metallic surface induced by machinery, J. Chin. Surf. Eng. 29 (2016) 6-16

14. L. Yang, The influence of nano-poillite lubricant additives on the abrasion performance of gray cast iron HT200, J. Sci. Mater. Eng. 40 (2010) 185-210

15. B.S. Xu, Microstructure, mechanical properties and tribological behaviour of tribofilm generated from natural serpentine mineral powders as lubricant additive, J. Sci. Wear 297 (2013) 802-810

16. X.W. Qi, Tribological properties of serpentine nanoparticles as oil additive under different material friction pairs, J. Sci. Adv. Mater. Res, 199 (2011) 1051-1057
17. Q.Y. Chang, Operando formation of an ultra-low friction boundary film from synthetic magnesium silicon hydroxide additive, J. Sci. Tribol. Int. 110 (2017) 35-40

18. Y.L. Yang, Fabrication of nanoserpentine-potassium acetate intercalation compound and its effect as additive on tribological properties of the fabric self-lubricating liner, J. Sci. Wear 318 (2014) 202-211

19. B.S. Xu, Sliding friction and wear behaviours of surfacecoated natural serpentine mineral powders as lubricant additive, J. Sci. Appl. Surf. Sci. 257 (2011) 2540-2549

20. B.S. Xu, Effect of thermal activation on the tribological behaviours of serpentine ultrafine powders as an additive in liquid paraffin, J. Sci. Tribol. Int. 44 (2011) 1736-1741

21. C. Yan, Tribological properties of serpentine, $\mathrm{La}(\mathrm{OH})_{3}$ and their composite particles as lubricant additives, J. Sci. Wear 288 (2012) 72-77

Cite this article as: Libo Wang, Yi Yang, Gang Yang, Guohong Shui, Mingxia Wu, Self-repairing and tribological behaviour of steel-steel friction pairs lubricated with an oil with magnesium silicate hydrosilicate as additive, Manufacturing Rev. 6, 1 (2019) 